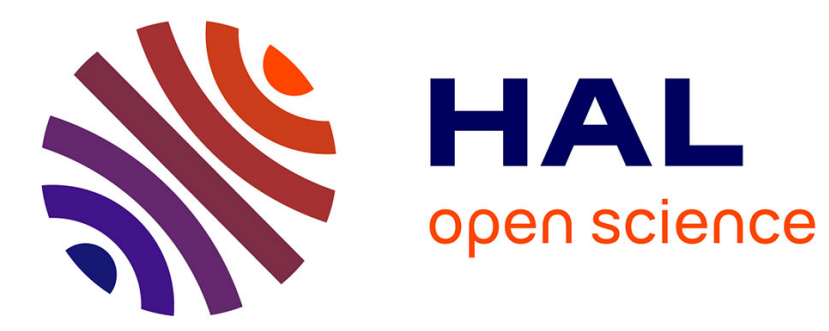

\title{
Discrimination des lupins à basse teneur en alcaloïdes par les adultes de Sitona lineatus L. (Col. Curculionidae)
}

Pierre Cantot, Jacques Papineau

\section{To cite this version:}

Pierre Cantot, Jacques Papineau. Discrimination des lupins à basse teneur en alcaloïdes par les adultes de Sitona lineatus L. (Col. Curculionidae). Agronomie, 1983, 3 (9), pp.937-940. hal-00884593

\section{HAL Id: hal-00884593 \\ https://hal.science/hal-00884593}

Submitted on 1 Jan 1983

HAL is a multi-disciplinary open access archive for the deposit and dissemination of scientific research documents, whether they are published or not. The documents may come from teaching and research institutions in France or abroad, or from public or private research centers.
L'archive ouverte pluridisciplinaire HAL, est destinée au dépôt et à la diffusion de documents scientifiques de niveau recherche, publiés ou non, émanant des établissements d'enseignement et de recherche français ou étrangers, des laboratoires publics ou privés. 


\title{
Discrimination des lupins à basse teneur en alcaloïdes par les adultes de Sitona lineatus L. (Col. Curculionidae)
}

\author{
Pierre CANTOT \& Jacques PAPINEAU $\left(^{*}\right)$ \\ I.N.R.A., Laboratoire de Zoologie, \\ (*) Station d'Amélioration des Plantes fourragères F 86600 Lusignan
}

\section{RÉSUMÉ}

\begin{abstract}
Dans le cadre du développement des cultures de lupin, la recherche s'oriente vers la sélection de types à basse teneur en alcaloides. Suite à des observations effectuées au champ, une expérimentation en conditions semicontrôlées a permis de démontrer que les adultes de Sitona lineatus L. s'alimentent préférentiellement sur les types pauvres en alcaloïdes alors qu'ils délaissent, même en alimentation forcée, les types plus riches. L'expérimentation a été effectuée avec des jeunes adultes ne s'étant jamais alimentés et la notation d'attaque est basée sur le nombre de denticulations réalisées par les insectes sur le bord du limbe des folioles des premières feuilles de différentes familles de Lupinus.

Ce type de sélection trophique présente un intérêt certain pour un tri rapide d'un grand nombre de plantes notamment lorsque les mutants à basse teneur sont rares.
\end{abstract}

Mots clés additionnels : Dégâts, Infestation artificielle, Plantules.

To develop lupin-growing, we have to breed cultivars with low alkaloid content. Following field observations, an experiment under semi-controlled conditions showed that adults of Sitona lineatus $\mathbf{L}$. fed preferentially on low-alkaloid plants and hardly at all on bitter plants. The experiment was carried out with newly emerged adults which had not fed previously.

Scoring of damage was based on the number of crescent-shape pieces eaten by the insects on the edge of the first leaflets of different Lupinus families. This kind of trophic selection is very useful for a quick screening of a great number of plants, especially when the types with low-alkaloid content are scarce, as in Lupinus mutabilis.

Additional key words : Damage, Artificial infestation, Seedlings.

\section{INTRODUCTION}

Les lupins ont toujours suscité de l'intérêt et leur utilisation remonte à l'Antiquité. Par contre, le développement des cultures a été limité par la présence de substances amères, les alcaloïdes, dans la plante et ses graines. Ce n'est qu'en 1927-30 que VON SENGBUSCH, découvrit les premiers mutants doux naturels. En 1974, LENOBLE démontrait l'intérêt du lupin blanc doux (Lupinus albus L.) en tant que plante protéagineuse (LENOBLE, 1983) et la recherche s'oriente actuellement vers la sélection de variétés à hauts rendements, résistantes à l'hiver ayant un grain de bonne qualité ne contenant pas d'alcaloides. Le rôle de ces substances dans la plante est complexe mais elles contribuent certainement à leur protection contre les ravageurs.
C'est ainsi que lièvres, lapins ou même gros bétail ne consomment que les plantes douces. KRZYMANSKA (1967) et WEGOREK \& KRZYMANSKA (1971) signalent le rôle des alcaloïdes dans la protection vis-à-vis du puceron vert du pois (Acyrthosiphon pisum Harr.). FORBES \& BECK (1954) indiquent que 2 espèces de thrips (Frankliniella tritici Firch et $F$. bispinosa Morgan) s'alimentent préférentiellement sur les plantes douces. Pour notre part, nous avons observé sur les cultures en plein champ que certains lupins avaient leurs feuilles dentelées. Ces encoches semi-circulaires sur le bord du limbe des folioles sont provoquées par l'adulte d'un coléoptère Curculionidae, Sitona lineatus $\mathrm{L}$. Cette espèce n'est pas inféodée au lupin mais elle s'en nourrit volontiers au stade adulte. Le développement larvaire s'effectue normalement sur le pois (Pisum sativum L.), la féverole 
(Vicia faba L.) ainsi que sur d'autres légumineuses annuelles spontanées. Le lupin héberge cette espèce soit au printemps après: la sortie des lieux d'hivernation soit en été lors de la migration des jeunes adultes provenant des cultures voisines.

L'examen de génotypes de lupins blancs attaqués par les sitones au champ nous a permis de constater que seuls les types doux étaient touchés et qu'en particulier la disjonction 3-1 dans la descendance $F_{2}$ d'hybrides amers-doux était très nette.

Pour vérifier ces observations faites au champ, nous avons réalisé une expérimentation en conditions semicontrôlées.

\section{MATÉRIEL ET MÉTHODES}

Des familles douces et amères de 3 espèces de lupin ( $L$. albus L., L. luteus L., L. mutabilis Sweet) ont été semécs, seules ou en mélange, dans des caissettes. Les caractéristiques de ces familles sont données dans le tableau 1. Pour $L$. albus, les 3 farnilles se différencient également par des

TABLEAU 1

Caractéristiques des familles.

Characteristics of famillies.

\begin{tabular}{llcl}
\hline Famille & Espèce & $\begin{array}{c}\text { Teneur \% } \\
\text { en alcaloides } \\
\text { du grain }\end{array}$ & Classe \\
\hline A1 & L. albus & $<0,02$ & Doux \\
A2 & L. albus & 0,1 & $1 / 2$ Amer \\
A3 & L. albus & 1,5 & Amer \\
L & L. Juteus & $<0,02$ & Doux \\
M1 M2 M3 L. mutabilis & 2,5 & Amer \\
\hline \hline
\end{tabular}

caractères marqueurs phénotypiques qui permettent de les identifier en mélange. Les différents traitements réalisés sont indiqués dans le tableau 2 ainsi que l'effectif total des plantes levées pour les 3 répétitions de chaque traitement. L'expérimentation a eu lieu en juillet à l'extérieur. Les jeunes adultes de sitones provenant d'un champ de pois ne se sont jamais alimentés et ont été mis en présence des plantules $20 \mathrm{j}$ après la levée, l'infestation ayant eu lieu sous cage à raison d'un adulte pour 3 plantes. L'intensité des dégâts occasionnés aux folioles a été notée 3 semaines plus tard selon l'échelle illustrée par la figure 1.

\section{RESULTATS}

A l'aide du test $\mathrm{X}^{2}$ nous avons mis en évidence qu'il n'y avait pas de différences significatives entre répétitions d'un même traitement. En présence de plantes douces et amères, les sitones choisissent préférentiellement les plantes douces, bien que les plantes amères présentent de faibles attaques (tabl. 3). C'est ainsi que la famille A1 dans les traitements T1 et T3 présente plus de 50 p. 100 des plantes avec la note 3 et 30 p. 100 avec la note 2 , alors que les familles amères n'ont jamais atteint la note 3 et très rarement la note 2.

Le traitement T4 montre qu'il n'y a pas de différence significative $\left(\mathrm{X}^{2}\right)$ entre la famille $\mathrm{A} 1$, qui possède le gène «pauper » $\left(^{*}\right)$ et la famille A2 qui a le gène « nutricius » $\left(^{*}\right)$ plus riche en alcaloïdes. Ceci tendrait à prouver qu'il y a un seuil de sensibilité.

Le traitement T2 montre que, malgré l'obligation de consommation sur des familles amères homogènes, les

$\left(^{*}\right)$ Gènes dont dépend l'absence d'alcaloïdes (HACKBARTH \& TROLL, 1959).
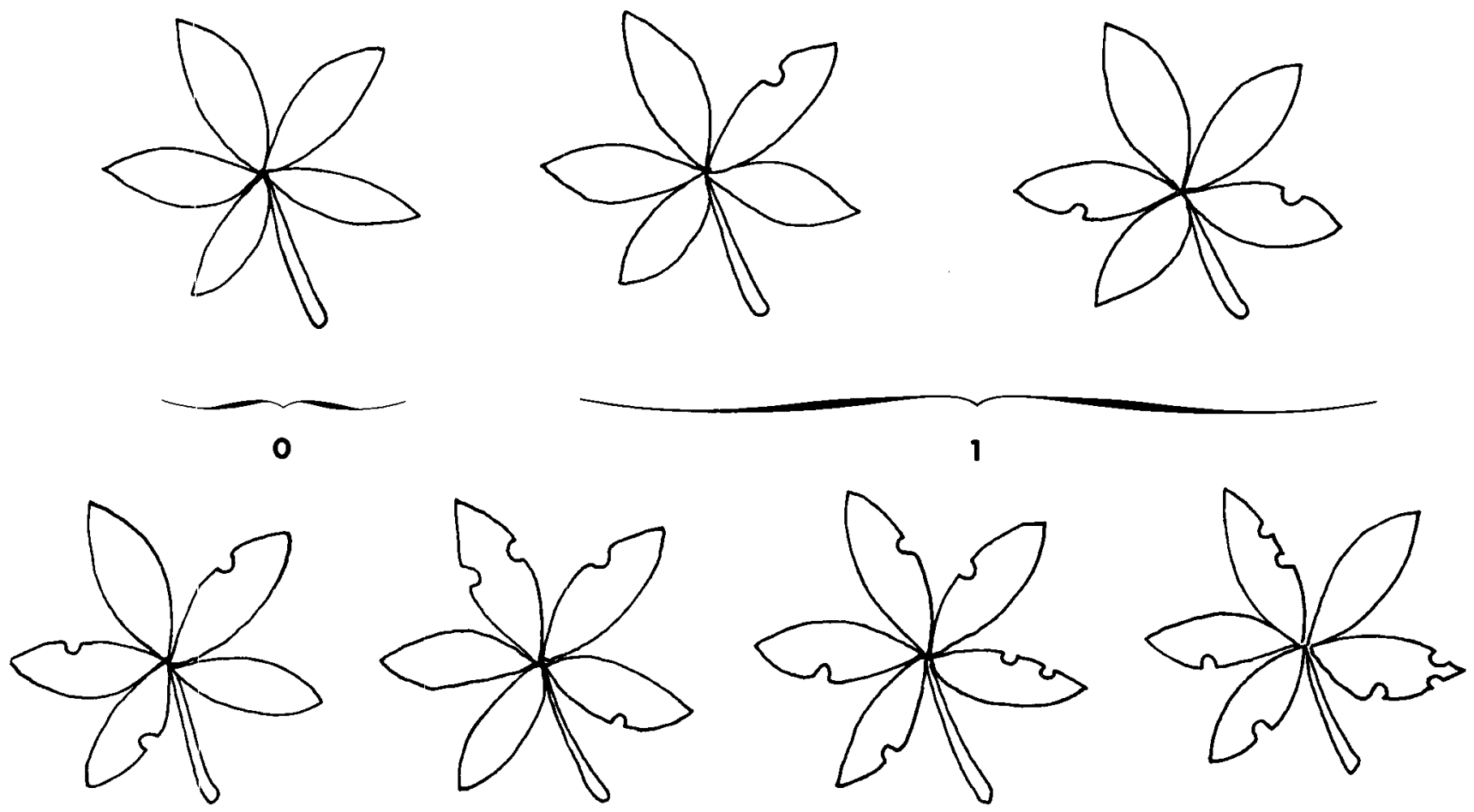

Figure 1

Echelle de notation sur la première feuille.

Assessment scale on the first leaf. 
TABLEAU 2

Description des différents traitements.

Description of treatments.

\begin{tabular}{|c|c|c|c|c|c|}
\hline \multirow{2}{*}{ Traitement } & \multirow{2}{*}{$\begin{array}{l}\text { Type } \\
\text { de } \\
\text { semis }\end{array}$} & \multirow{2}{*}{ Familles } & \multicolumn{3}{|c|}{ Effectifs selon les classes } \\
\hline & & & Doux & 1/2 Amer & Amers \\
\hline 1 & mélange & $\mathrm{A} 1, \mathrm{~L}, \mathrm{~A} 3, \mathrm{M} 1$ & $15+16$ & & $14+14$ \\
\hline 2 & tous amers & $\mathrm{M} 1, \mathrm{M} 2, \mathrm{M} 3$ & & & $18+15+19$ \\
\hline 3 & mélange & $\mathrm{A} 1, \mathrm{~A} 3$ & 14 & & 14 \\
\hline 4 & mélange & $\mathrm{A} 1, \mathrm{~A} 2, \mathrm{~A} 3$ & 19 & 20 & 19 \\
\hline
\end{tabular}

TABLEAU 3

Fréquence (en p. cent) des différentes notes d'attaques selon les familles et les traitements.

Frequency (in \%) of attack scores attributed to different families and treatments.

\begin{tabular}{|c|c|c|c|c|c|c|c|c|c|}
\hline \multirow{2}{*}{$\begin{array}{l}\text { Famille } \\
\text { de lupin }\end{array}$} & \multicolumn{4}{|c|}{ Notation } & \multirow{2}{*}{$\begin{array}{l}\text { Famille } \\
\text { de lupin }\end{array}$} & \multicolumn{4}{|c|}{ Notation } \\
\hline & 0 & 1 & 2 & 3 & & 0 & 1 & 2 & 3 \\
\hline $\mathrm{A} 1$ & 0 & 7 & 40 & 53 & M1 & 94 & 6 & 0 & 0 \\
\hline L & 0 & 0 & 19 & 81 & M2 & 73 & 20 & 7 & 0 \\
\hline $\mathrm{A} 3$ & 43 & 57 & 0 & 0 & M3 & 53 & 37 & 10 & 0 \\
\hline M1 & 36 & 43 & 21 & 0 & & & & & \\
\hline
\end{tabular}

Traitement 1

Traitement 2

\begin{tabular}{|c|c|c|c|c|c|c|c|c|c|}
\hline \multirow{2}{*}{$\begin{array}{l}\text { Famille } \\
\text { de lupin }\end{array}$} & \multicolumn{4}{|c|}{ Notation } & \multirow{2}{*}{$\begin{array}{l}\text { Famille } \\
\text { de lupin }\end{array}$} & \multicolumn{4}{|c|}{ Notation } \\
\hline & 0 & 1 & 2 & 3 & & 0 & 1 & 2 & 3 \\
\hline A1 & 7 & 7 & 29 & 57 & A1 & 0 & 26 & 47 & 26 \\
\hline \multirow[t]{2}{*}{ A3 } & 43 & 50 & 7 & 0 & $\mathrm{~A} 2$ & 0 & 35 & 40 & 25 \\
\hline & & & & & A3 & 0 & 68 & 32 & 0 \\
\hline
\end{tabular}

Traitement 3

Traitement 4

dégâts ne sont pas plus importants que ceux observés en mélange avec une famille douce.

Sur les familles amères, les attaques de sitone, lorsqu'elles existent, se situent toujours sur les feuilles les plus vieilles.

\section{DISCUSSION}

Il est généralement admis (NowACKI, 1963) que la teneur en alcaloides des lupins diminue au cours de la germination et de la phase juvénile avant de croître lorsque la photosynthèse prend le relais des organes de réserve.

Notre expérience se situe à un stade relativement jeune et pourtant les sitones font remarquablement la différence entre familles douces et amères. On peut supposer que les rares attaques de plantes issues de graines amères s'expliquent par une teneur faible en alcaloïdes due au stade des plantules, ce qui semble être confirmé par la localisation de ces attaques ( $1^{\text {res }}$ feuilles). Ce type de sélectivité trophique des insectes vis-à-vis de plantes contenant des substances antinutritionnelles n'est pas nouveau et plusieurs mises au point ont été publiées récemment (WALLACE \& MANSELL, 1976 ; Robinson, 1979). En ce qui concerne $S$. lineatus, des travaux ont été réalisés vis-à-vis de la résistance du pois à cet insecte (WNUK \& WIECH, 1980 ; AULD et al., 1980). Ce dernier auteur notamment utilise un test de routine dont le type de notation se rapproche du nôtre. Il faut dire qu'il s'agit là d'une démarche différente puisque l'insecte est dommageable pour la culture, ce qui n'est pas le cas pour $S$. lineatus dans le cas du lupin. Pour nous l'objectif est différent puisque ce sont les plantes les plus attaquées, donc moins riches en alcaloïdes, qui doivent retenir notre attention.

$\mathrm{Ce}$ test biologique rapide pourrait être utilisé pour repérer les plantes douces dans les familles en disjonction, mais surtout pour cribler un grand nombre de plantes lorsque la fréquence des plantes douces est très faible: mutagénèse, recherche de mutants naturels dans les différentes espèces etc...

Ce test pourrait être également d'une grande utilité pour la sélection de génotypes faibles en alcaloïdes lorsque le mutant doux n'existe pas encore, comme dans l'espèce $L$. mutabilis par exemple.

\section{REMERCIEMENTS}

Nous tenons à remercier R. BOURNOVILLF et M. LINNOBLt (I.N.R.A., Lusignan) pour leurs suggestions et critiques lors de l'élaboration de cette note.

Reçu le 3 mars 1983.

Accepté lè 16 mai 1983. 


\section{RÉFÉRENCES BIBLIOGRAPHIQUES}

Auld D. L., O'Keefe L. E., Murray G. A., Smith J. H., 1980. Diallel analyses of resistance to the adult pea leaf weevil in peas. Crop Sci., 20, 760-766.

Forbes I., Beck E. W., 1954. A rapid biological technique for screening blue lupin populations for low alkaloid plants. Agron. J., 46, $11,528-529$.

Hackbarth J., Troll H. J., 1959. Lupinen als Körnerleguminosen und Futterplanzen. Hand. der Planzenzücht., 4, 1-51.

Krzymanska J., 1967. The role of alkaloids in the resistance of some lupin varieties to the pea aphid. Biul. Inst. Ochr. Rosl., 36, 237-245.

Lenoble M., 1983. Criteria and methods in the selection of $L$. albus in "Perspectives for peas and lupins as protein crops $»$, p. 174-182.

$\mathrm{R}$. Thompson and R. Casey editors.
Nowacki E., 1963. Inheritance and biosynthesis of alkaloids in lupin. Genet. Pol., 4, 161-202.

Robinson T., 1979. The evolutionary ecology of alkaloids in herbivores. Their interaction with secondary plant metabolites. Rosenthal G. A. et Janzen D. ed., Academic Press inc., 657-682. Wallace J.W., Mansell R. L., 1976. Biochemical interaction between plants and insects in Recent Advances in Phytochemistry, vol. 10, Plenum press ed., $425 \mathrm{p}$.

Wegorek W., Krzymanska J., 1971. Further studies on the resistance of lupin to the pea aphid (Acyrthosiphon pisum Harris) Prace Nauk. Inst. Ochr. Rosl., 13, 7-23.

Wnuk A., Wiech K., 1980. Variétés de pois sensibles à l'activité alimentaire des adultes du genre Sitona. Pol. Pismo entomol., 50, 597-605.

I.N. 1.036 .007 .1 